ARTICLE

Received 6 Aug 2015 | Accepted 22 Mar 2016 | Published 29 Apr 2016

DOI: $10.1038 /$ ncomms11411

\title{
Mode engineering for realistic quantum-enhanced interferometry
}

\author{
Michał Jachura ${ }^{1}$, Radosław Chrapkiewicz ${ }^{1}$, Rafał Demkowicz-Dobrzański ${ }^{1}$, Wojciech Wasilewski ${ }^{1}$ \\ \& Konrad Banaszek ${ }^{1}$
}

Quantum metrology overcomes standard precision limits by exploiting collective quantum superpositions of physical systems used for sensing, with the prominent example of non-classical multiphoton states improving interferometric techniques. Practical quantum-enhanced interferometry is, however, vulnerable to imperfections such as partial distinguishability of interfering photons. Here we introduce a method where appropriate design of the modal structure of input photons can alleviate deleterious effects caused by another, experimentally inaccessible degree of freedom. This result is accompanied by a laboratory demonstration that a suitable choice of spatial modes combined with position-resolved coincidence detection restores entanglement-enhanced precision in the full operating range of a realistic two-photon Mach-Zehnder interferometer, specifically around a point which otherwise does not even attain the shot-noise limit due to the presence of residual distinguishing information in the spectral degree of freedom. Our method highlights the potential of engineering multimode physical systems in metrologic applications.

\footnotetext{
${ }^{1}$ Faculty of Physics, University of Warsaw, Pasteura 5, 02-093 Warsaw, Poland. Correspondence and requests for materials should be addressed to R.C. (email: radekch@fuw.edu.pl).
} 
Q uantum phenomena can facilitate and boost the performance of imaging techniques ${ }^{1-4}$, sensitive measurements in delicate materials ${ }^{5,6}$, as well as detection schemes probing subtle physical effects such as gravitational waves ${ }^{7}$. These strategies rely on preparing collective superposition states of multiple probes (for example, photons, atoms) to achieve precision enhancement beyond standard limits ${ }^{8-17}$. In the optical domain, a common strategy for collective state preparation is to realize multiphoton interference in linear circuits, for example, free space or integrated interferometers ${ }^{18-21}$, fed with non-classical states of light. Attainable precision can be, however, markedly vulnerable to residual distinguishing information between interfering photons. Standard methods to improve indistinguishability based on filtering are often inadequate, in particular, introducing attenuation that may easily diminish the overall benefit of collective state preparation.

The purpose of this paper is to analyse the interplay between degrees of freedom with different experimental accessibility in two-photon interferometry, which is a canonical example of a quantum-enhanced measurement ${ }^{22}$. We demonstrate that detrimental effects caused by distinguishing information present in one degree of freedom that is beyond experimental control or lacks technical means to improve indistinguishability, can be alleviated by mode engineering in another degree of freedom, even though these two remain completely uncorrelated. This feature is investigated in the case of local phase estimation, whose precision becomes strongly dependent on the operating point if the two photons feeding the interferometer exhibit residual distinguishability. It is shown that a carefully designed preparation and detection scheme for a degree of freedom other than the one causing distinguishability allows one to restore quantum-enhanced precision in the entire operating range of the interferometer. We attribute this effect to non-trivial combination of one- and two-photon interference that turns out to augment phase sensitivity beyond the shot-noise limit. We also present an example indicating that similar enhancement occurs also at higher photon numbers. Prospectively, the results reported here may provide another class of strategies to mitigate effects of imperfections and environmental noise in quantum-enhanced metrology $23-29$. The theoretical analysis is complemented with an experiment investigating sensitivity of a balanced Mach-Zehnder interferometer fed with photon pairs. We determine the precision of local phase estimation around the operating point when the photons coalesce pairwise at the interferometer output ports. In this regime, the residual spectral distinguishability within pairs has a markedly deleterious effect on the attainable precision. Building on recent advances in spatially resolved single-photon detection $^{1,30-39}$, we demonstrate that by controlling the input spatial structure of interfering photons and extracting complete spatial information at the detection stage it is nevertheless possible to recover the sub-shot-noise precision. This confirms in proof-of-principle settings the feasibility of mode engineering techniques for quantum-enhanced interferometry.

\section{Results}

Realistic two-photon interferometer. A generic two-photon Mach-Zehnder interferometer constructed with a pair of balanced 50/50 beam splitters and fed with photon pairs is shown schematically in Fig. 1a. The phase shift $\theta$ between the interferometer arms modulates probabilities of detection events at the output ports that can be grouped into two types: either the photons exit through different paths, producing a coincidence event between the detectors monitoring the ports, or both are found in the same output port leading to a double event. If the two photons are indistinguishable at the input, the first beam splitter generates a coherent superposition of both the photons in one or another arm of the interferometer, which is the simplest case of a N00N state providing sensitivity that approaches the Heisenberg limit ${ }^{10-12}$.

In the multimode description of the setup one introduces two sets of annihilation operators for upper path modes $\hat{a}_{\mu}$ and lower path modes $\hat{b}_{\mu}$ that are matched pairwise. Individual modes in each arm are mutually orthogonal, that is, $\left[\hat{a}_{\mu}, \hat{a}_{v}^{\dagger}\right]=\left[\hat{b}_{\mu}, \hat{b}_{v}^{\dagger}\right]=\delta_{\mu v}$. The unitary map $\hat{U}(\theta)$ implemented by the interferometer between the input and the output ports transforms pairs of field operators labelled with the same index $\mu$ as

$$
\hat{U}^{\dagger}(\theta)\left(\begin{array}{l}
\hat{a}_{\mu} \\
\hat{b}_{\mu}
\end{array}\right) \hat{U}(\theta)=\left(\begin{array}{cc}
\cos \frac{\theta}{2} & \sin \frac{\theta}{2} \\
\sin \frac{\theta}{2} & -\cos \frac{\theta}{2}
\end{array}\right)\left(\begin{array}{l}
\hat{a}_{\mu} \\
\hat{b}_{\mu}
\end{array}\right) .
$$

Partial distinguishability of the interfering photons can be modelled by assuming that at the input the photon in the upper path occupies a certain mode $\hat{a}_{1}$, while the lower path photon is prepared in a combination of a matching mode $\hat{b}_{1}$ and another orthogonal mode $\hat{b}_{2}$ with relative weights $\mathcal{V}$ and $1-\mathcal{V}$, where $\mathcal{V}$ is the visibility parameter specifying the fraction of indistinguishable pairs. To keep the notation concise, we will write the complete two-photon state as pure

$$
|\psi\rangle=\hat{a}_{1}^{\dagger}\left(\sqrt{\mathcal{V}} \hat{b}_{1}^{\dagger}+\sqrt{1-\mathcal{V}} \hat{b}_{2}^{\dagger}\right)|\mathrm{vac}\rangle
$$

and trace the final formulas over the index $\mu=1,2$ for the initial modes $\hat{b}_{\mu}$. This is equivalent to taking from the start a reduced density matrix $\mathcal{V} \hat{a}_{1}^{\dagger} \hat{b}_{1}^{\dagger}|\operatorname{vac}\rangle\left\langle\operatorname{vac}\left|\hat{a}_{1} \hat{b}_{1}+(1-\mathcal{V}) \hat{a}_{1}^{\dagger} \hat{b}_{2}^{\dagger}\right| \operatorname{vac}\right\rangle\langle\operatorname{vac}| \hat{a}_{1} \hat{b}_{2}$. Here $|v a c\rangle$ is the vacuum state of the entire multimode electromagnetic field satisfying $\hat{a}_{\mu}|\mathrm{vac}\rangle=\hat{b}_{\mu}|\mathrm{vac}\rangle=0$ for any $\mu$. Note that the above model includes the case of partly overlapping wavepackets constructed from a continuum of modes, wherein $\hat{b}_{1}$ and $\hat{b}_{2}$ can be identified through the standard algebraic technique of Gram-Schmidt orthogonalization.

The general transformation from equation (1) taken with $\mu=1,2$ implies the following expression for the probability of a coincidence event

$$
p_{c}(\theta)=1-\frac{1}{2}(1+\mathcal{V}) \sin ^{2} \theta,
$$

while the probability of a double event is $p_{d}(\theta)=1-p_{c}(\theta)$. These formulas combine expressions for fully indistinguishable and completely distinguishable pairs with respective probabilities $\mathcal{V}$ and $1-\mathcal{V}$. The resulting fringes are depicted in Fig. $1 \mathrm{~b}$ for $\mathcal{V}=1$ and 0.93 .

As a consequence of the Cramér-Rao bound ${ }^{40}$, the minimum uncertainty of any unbiased phase estimate obtained from a measurement using $N$ photon pairs around an operating point $\theta$ is given by

$$
\Delta^{\text {pair }}=\frac{1}{\sqrt{N F^{\text {pair }}(\theta)}},
$$

where for standard photon counting at output ports of the interferometer the Fisher information $F^{\text {pair }}(\theta)$ is given by a sum of two terms contributed by coincidence and double events ${ }^{17}$,

$$
F^{\text {pair }}(\theta)=\frac{1}{p_{\mathrm{c}}(\theta)}\left(\frac{\mathrm{d} p_{\mathrm{c}}}{\mathrm{d} \theta}\right)^{2}+\frac{1}{p_{\mathrm{d}}(\theta)}\left(\frac{\mathrm{d} p_{\mathrm{d}}}{\mathrm{d} \theta}\right)^{2} .
$$

As a reference, we will take the uncertainty of ideal, shot-noiselimited phase measurement $\Delta^{\text {shot }}=1 / \sqrt{2 N}$, when $2 N$ photons are sent individually to the interferometer. Our figure of merit will be the ratio $\varepsilon=\Delta^{\text {pair }} / \Delta^{\text {shot }}$ of these two uncertainties, with $\varepsilon<1$ implying that sub-shot-noise precision has been achieved. 


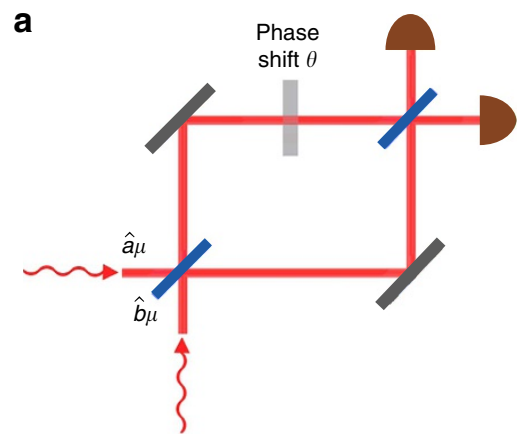
- Two photon interference $\mathcal{V}=1$
--n-. Two photon interference $\mathcal{V}=0.93$
--r-- Single-photon interference

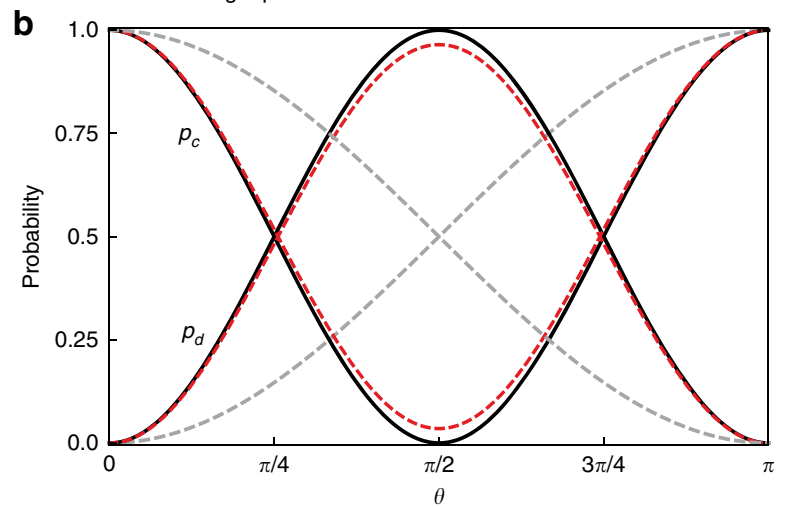

C

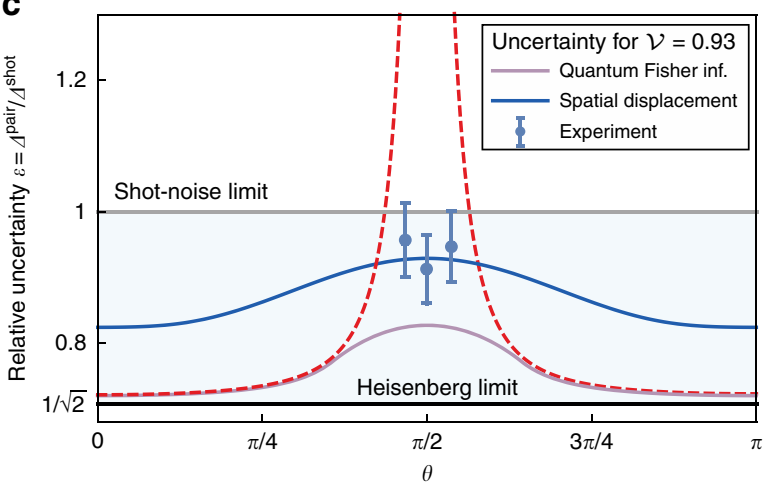

Figure 1 | Two-photon interferometer. (a) In the multimode description, two paths are described by families of annihilation operators $\hat{a}_{\mu}$ and $\hat{b}_{\mu}$ subjected pairwise to a unitary map dependent on the phase shift $\theta$. (b) Probabilities of coincidence $p_{c}$ and double count events $p_{d}$ at the interferometer output are noticeably affected around $\theta \approx \pi / 2$ by imperfect indistinguishability (red dashed lines for the visibility $\mathcal{V}=0.93$ ) when compared with the ideal case (black solid lines). Grey dashed lines depict standard single-photon interference fringes, when only one input port is illuminated. (c) Residual distinguishability has a dramatic effect on the phase estimation uncertainty, shown with correspondingly coded lines, which diverges at $\theta=\pi / 2$ for non-unit visibility $\mathcal{V}$. Engineering overlap in an additional degree of freedom of the interfering photons and implementing optimal measurement allows one to restore the sub-shot-noise precision over the entire operating range, shown with the purple solid line when the fraction of overlapping pairs is optimized individually for each operating point. The solid grey line depicts the shot-noise limit of relative uncertainty. The blue solid line depicts an explicit strategy based on introducing a fixed transverse displacement between interferometer inputs and performing spatially resolved detection. These predictions are confirmed by the experimentally determined estimation precision, depicted as solid circles with two s.d.s. error bars.
When the interferometer is fed with pairs of perfectly indistinguishable photons characterized by $\mathcal{V}=1$, we have $\varepsilon=1 / \sqrt{2}$ independently of the operating point of the interferometer. It can be verified that around $\theta=0$ (equivalent to $\theta=\pi$ ) and $\theta=\pi / 2$ the main contribution to Fisher information defined in equation (5) comes, respectively, from double or coincidence events that occur with vanishing probabilities when approaching these phase values. This is because in the ideal scenario even a small number of rare events provides a sound basis to infer the phase shift. Such a regime corresponds in standard interferometry to dark-fringe operation used, for example, in gravitational wave detectors ${ }^{7,41}$.

As seen in Fig. 1c, the precision of phase estimation is affected markedly by the non-ideal indistinguishability of photon pairs. In particular, statistical noise generated by non-vanishing background of coincidence events effectively suppresses information about the phase shift that could be retrieved around $\theta=\pi / 2$. We will refer to this operating point as the coincidence dark fringe. An analogous effect would be observed also at $\theta=0$ if any mechanism generating spurious double events was incorporated into calculations.

Restoring quantum enhancement. The analysis presented above assumed that we have no access to the degree of freedom introducing partial distinguishability. For concreteness, we will consider this degree of freedom to be the spectral one, which means that all measurements performed on the photons are integrated in the frequency domain. Suppose now that we can fully control and measure another, uncorrelated degree of freedom of the photon pairs sent to the interferometer. For the clarity of the argument, it will be convenient to use in this role the transverse spatial characteristics of the photons. Let us consider a scenario when in addition to spectral distinguishability characterized by $\mathcal{V}$ we reduce the spatial overlap of the photons by preparing them in nonorthogonal spatial modes. As a result, even in the regime of perfect spectral indistinguishability only a fraction $\mathcal{D}$ of photon pairs would effectively overlap in space. To account for this scenario we will take the mode index to have two components $\mu=i \chi$, where $i=1,2$ refers to the spectral degree of freedom, while $\chi=R, L$ denotes two mutually orthogonal spatial modes. Using this notation, the input state $\left|\psi_{\mathcal{D}}\right\rangle$ is described by an expression analogous to equation (2) with the following substitution of creation operators:

$$
\begin{aligned}
& \hat{a}_{1}^{\dagger} \rightarrow \hat{a}_{1 R}^{\dagger} \\
& \hat{b}_{i}^{\dagger} \rightarrow \sqrt{\mathcal{D}} \hat{b}_{i R}^{\dagger}+\sqrt{1-\mathcal{D}} \hat{b}_{i L}^{\dagger}, \quad i=1,2
\end{aligned}
$$

and it explicitly reads

$$
\begin{aligned}
\left|\psi_{\mathcal{D}}\right\rangle= & \hat{a}_{1 R}^{\dagger}\left[\sqrt{\mathcal{V}}\left(\sqrt{\mathcal{D}} \hat{b}_{1 R}^{\dagger}+\sqrt{1-\mathcal{D}} \hat{b}_{1 L}^{\dagger}\right)\right. \\
& \left.+\sqrt{1-\mathcal{V}}\left(\sqrt{\mathcal{D}} \hat{b}_{2 R}^{\dagger}+\sqrt{1-\mathcal{D}} \hat{b}_{2 L}^{\dagger}\right)\right]|\mathrm{vac}\rangle
\end{aligned}
$$

Note that both the spectral components $\hat{b}_{1}$ and $\hat{b}_{2}$ have been subjected to the same spatial transformation. This is in accordance with our assumption that photon manipulations cannot depend on the inaccessible spectral degree of freedom.

Although the spectral and the spatial degrees of freedom are treated on equal footing in equation (7), the crucial difference is the ability to measure the latter at the interferometer output. Therefore, the phase shift $\theta$ can be read out from the paths taken by the photons as well as their transverse spatial properties. To find the optimal strategy, we will resort to the concept of quantum Fisher information $F_{\mathrm{Q}}(\theta)$ (refs 42,43), which defines through an expression analogous to equation (4), the minimum uncertainty of a phase estimate inferred from the entire available 
characteristics of the physical system used for sensing. When the spatial degree of freedom for coincidence events is taken into account, the explicit expression for quantum Fisher information at the coincidence dark fringe $\theta=\pi / 2$, where the effects of spectral distinguishability are most severe, reads

$$
F_{\mathrm{Q}}(\pi / 2)=2 \frac{1-\mathcal{D}^{2}}{1-\mathcal{D} \mathcal{V}} .
$$

Detailed derivation of this result is presented in the Methods section. For a given $\mathcal{V}$, the maximum value of the above expression is obtained for $\mathcal{D}^{\text {opt }}=\left(1-\sqrt{1-\mathcal{V}^{2}}\right) / \mathcal{V}$ and reads $F_{\mathrm{Q}}^{\mathrm{opt}}(\pi / 2)=4\left(1-\sqrt{1-\mathcal{V}^{2}}\right) / \mathcal{V}^{2}$. Remarkably, this value gives sub-shot-noise precision for any $\mathcal{V}>0$. In Fig. 2, we compare the precision implied by numerically computed $F_{Q}(\theta)$ in the range $0 \leq \theta \leq \pi$ with $\mathcal{D}$ optimized for an individual operating point to a scenario when no mode engineering has been attempted. A cross-section of these plots for $\mathcal{V}=0.93$ has also been shown in

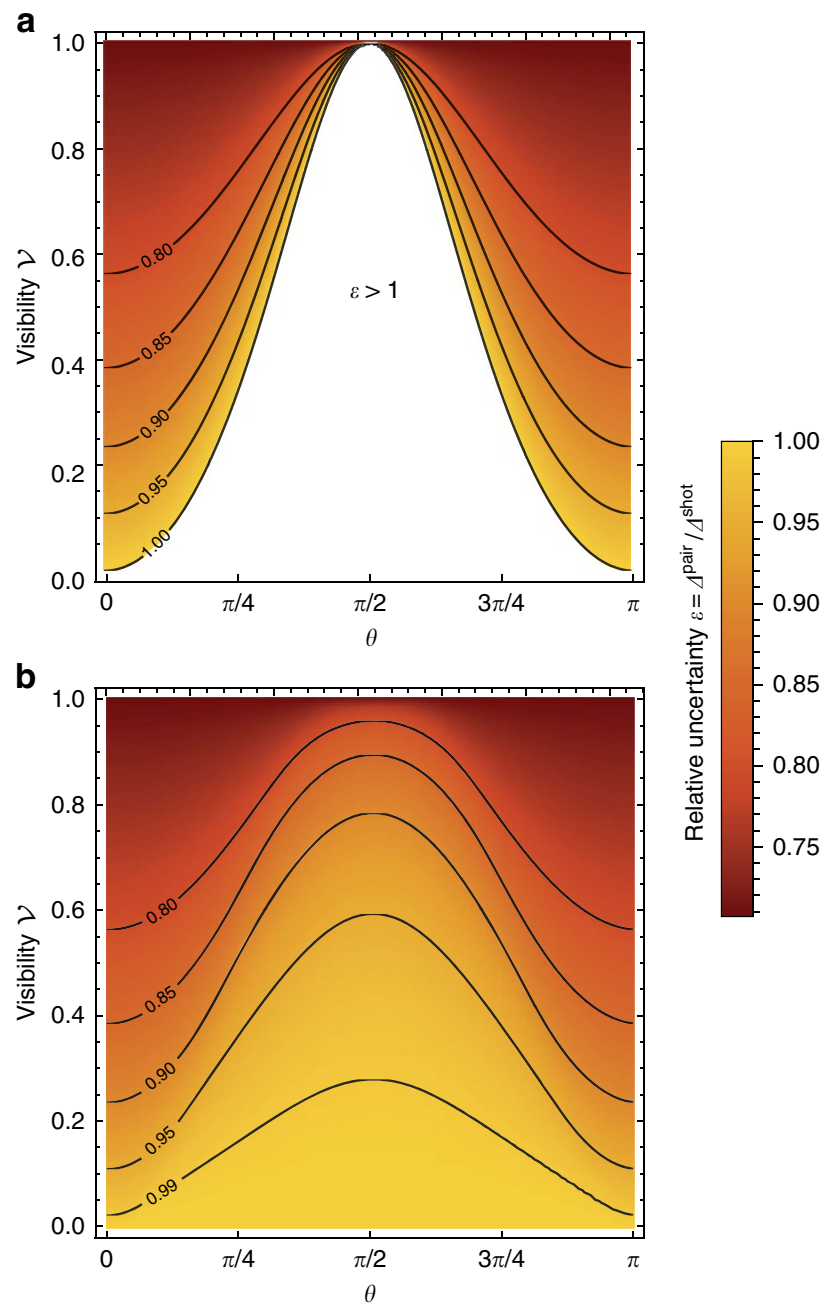

Figure 2 | Enhancement of estimation precision. Relative enhancement $\varepsilon=\Delta^{\text {pair }} / \Delta^{\text {shot }}$ for (a) full spatial overlap $\mathcal{D}=1$ of the two input photons and (b) the overlap parameter $\mathcal{D}$ optimized individually for each given spectral visibility $0 \leq \mathcal{V} \leq 1$ and an operating point $0 \leq \theta \leq \pi$. The white area in a depicts the region $\varepsilon>1$ where sub-shot-noise sensitivity is lost. It is seen that spatial mode engineering allows one to restore quantum enhancement across the entire parameter range. The uncertainty of the two-photon scheme $\Delta^{\text {pair }}$ is given by quantum Fisher information derived in equation (26).
Fig. 1c. It is seen that the singularity in precision around the coincidence dark fringe is removed and that the sub-shot-noise operation is ensured across the entire range of $\theta$. Note that this result is achieved without any post-selection of two-photon detection events and no filtering or any other manipulation in the spectral domain has been applied.

The standard strategy to improve spectral indistinguishability in two-photon experiments is to restrict the bandwidth of detected photons using narrowband interference filters. However, for any finite filtering bandwidth the visibility parameter would remain below one and consequently the singularity in the uncertainty of phase estimation around $\theta=\pi / 2$ would not be removed. Furthermore, filtering reduces the number of detection events that can be used for estimation. Starting from a simple model for partial distinguishability based on gaussian spectral profiles, we verified that if the number of input photon pairs is taken as a benchmark to calculate the relative uncertainty, spectral filtering does not improve the measurement precision at all.

Measurement scheme. To elucidate the origin of the above effect, it is instructive to analyse the operation of the interferometer in the $R / L$ basis of transverse spatial modes. Detecting both the photons in $R R$ modes means that they overlapped spatially at the input and therefore underwent imperfect two-photon interference affected by non-unit spectral visibility $\mathcal{V}$ with fringes shown in Fig. 1b. On the other hand, combinations $R L$ and $L R$ at the output imply that the upper path photon was initially in the mode $R$ and the lower path photon in the mode $L$. Consequently, both the photons propagated through the interferometer as independent particles exhibiting single-photon interference, which for $\theta=\pi / 2$ gives the steepest slope of interference fringes as seen in Fig. 1b. If the lower path photon was prepared in a statistical mixture of $R$ and $L$ modes, only single-photon interference would provide information about the phase shift at $\theta=\pi / 2$ and the shot-noise limit could not be surpassed. However, because the lower path photon is sent into the interferometer in a superposition of the modes $R$ and $L$, information from two- and one-photon interference can be combined in a coherent way through a suitable choice of the measurement basis at the interferometer output. Strikingly, although neither one- nor two-photon interference used separately beats the shot-noise limit itself, their coherent combination restores quantum enhancement of the measurement.

As derived in the Methods section, the explicit form of the optimal measurement attaining quantum Fisher information at the coincidence dark fringe requires discrimination between double events and two types of coincidence events corresponding to the following projections in the spatial degree of freedom:

$$
\begin{aligned}
| \pm\rangle=\sqrt{\frac{\mathcal{D}}{1+\mathcal{D}}}|R R\rangle & +\frac{1}{2}\left(\sqrt{\frac{1-\mathcal{D}}{1+\mathcal{D}}} \pm 1\right)|R L\rangle \\
& +\frac{1}{2}\left(\sqrt{\left.\frac{1-\mathcal{D}}{1+\mathcal{D}} \mp 1\right)|L R\rangle}\right.
\end{aligned}
$$

In Fig. 3, we depict the resulting interference fringes for all three types of events when $\mathcal{D}$ is optimized for $\mathcal{V}=93 \%$ at the operating point $\theta=\pi / 2$. It is seen that coincidence events resolved in the \pm basis indeed exhibit both one- and two-photon interference providing a rather steep slope at $\theta=\pi / 2$, while their overall probability remains relatively low at this operating point. Combination of these features yields sub-shot-noise sensitivity.

In the limit $\mathcal{V} \rightarrow 0$ the optimal $\mathcal{D}$ approaches zero, which means that at the input the two photons are nearly fully 


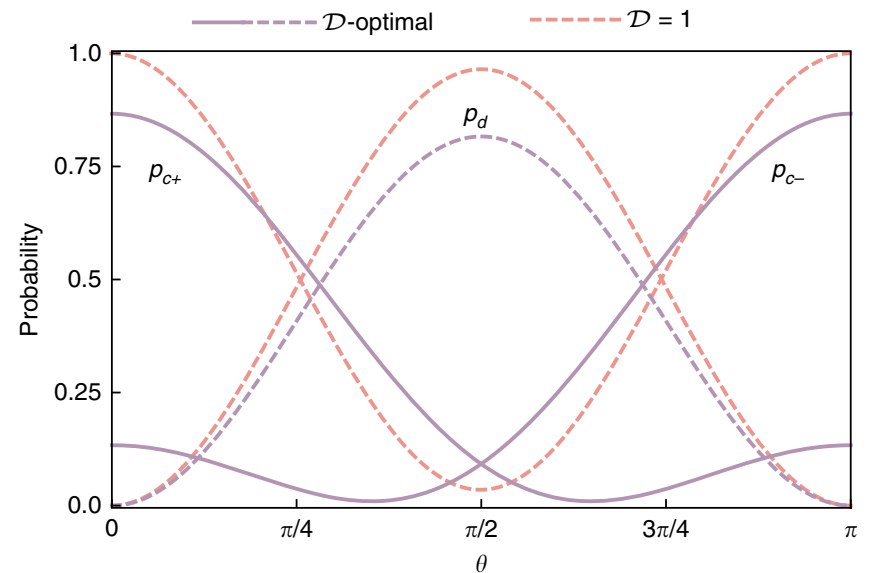

Figure 3 | Optimized interference fringes. The probabilities of double $p_{d}$ (purple dashed line) and two types of coincidence $p_{\mathrm{c}} \pm$ (purple solid lines) events corresponding to projections in the optimal basis $| \pm\rangle$ defined in equation (9) with the overlap parameter $\mathcal{D}$ optimizing quantum Fisher information at $\theta=\pi / 2$ for the visibility $\mathcal{V}=0.93$ in the inaccessible degree of freedom. Dashed red lines show standard two-photon interference fringes for the same visibility without engineering an additional degree of freedom.

distinguishable in their spatial degree of freedom. In this regime the asymptotic expressions for the optimal measurement basis are $|+\rangle=|R L\rangle$ and $|-\rangle=|L R\rangle$, that is, we need to identify the origin of a photon that has appeared at a given output port. This is equivalent to realizing single-photon interference twice, each time sending a photon into a different input port. The above analysis explains why in the limit of zero visibility $\mathcal{V}=0$ we recover the shot-noise limit as evidenced by Fig. 2b. The described scheme is able to exploit non-classical two-photon interference for any $\mathcal{V}>0$ to achieve sub-shot-noise operation, although unsurprisingly with a diminishing quantum enhancement when $\mathcal{V} \rightarrow 0$.

Spatially resolved detection. The measurement maximizing quantum Fisher information requires implementation of rather exotic projections on two-photon superposition states given in equation (9). This leads to the question about practical realization of the measurement achieving sub-shot-noise precision at a given operating point. Fortunately, as we will now demonstrate, for generic spatial modes sub-shot-noise precision can be restored just by measuring transverse positions of photons emerging from the interferometer. To discuss quantitatively this idea it will be convenient to resort to the paraxial approximation and to introduce two sets of operators $\hat{a}_{i}(x)$ and $\hat{b}_{i}(x)$ labelled with a continuous one-dimensional transverse position $x$. As before, the index $i=1,2$ labels spectral modes. Suppose now that the photons entering the interferometer along the upper and the lower path are prepared in spatial modes described by respective normalized profiles $u(x)$ and $v(x)$,

$$
\begin{aligned}
\hat{a}_{i R} & =\int \mathrm{d} x u(x) \hat{a}_{i}(x) \\
\sqrt{\mathcal{D}} \hat{b}_{i R}^{\dagger}+\sqrt{1-\mathcal{D}} \hat{b}_{i L}^{\dagger} & =\int \mathrm{d} x v(x) \hat{b}_{i}(x) .
\end{aligned}
$$

The overlap parameter now reads $\mathcal{D}=\left|\int \mathrm{d} x u^{*}(x) v(x)\right|^{2}$. The probability of detecting photons in two different output ports at

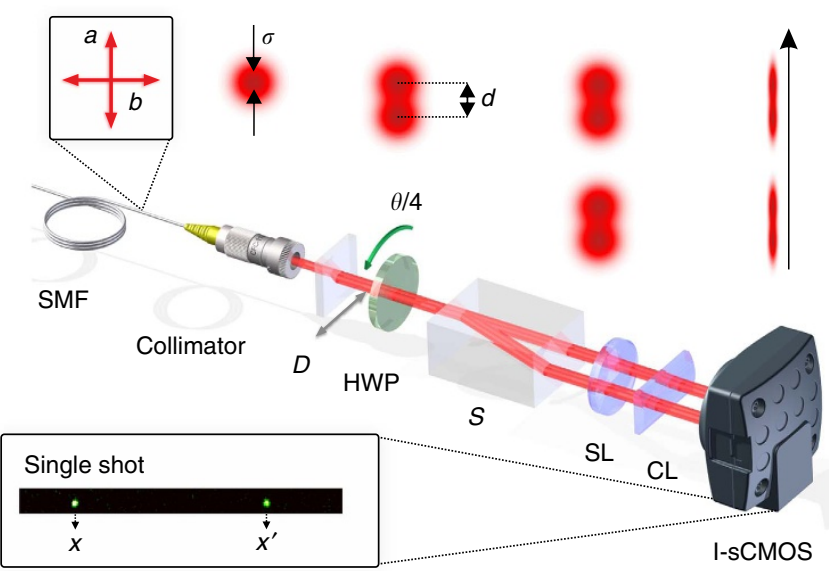

Figure 4 | Experimental setup. Interference between two orthogonally polarized photons $a$ and $b$ delivered by the single-mode fibre (SMF) is realized in the common path configuration with the phase shift $\theta$ corresponding to the quadrupled rotation angle of the half-wave plate (HWP). The preceding calcite displacer $D$ introduces transverse displacement between photon modes, which combined with spatially resolved detection restores the sub-shot-noise precision of phase estimation around the coincidence dark fringe. The output ports of the calcite beam separator $S$ are mapped using a spherical lens $(\mathrm{SL})$ and a cylindrical lens $(\mathrm{CL})$ onto the intensified SCMOS camera detecting individual coincidence events with spatial resolution as shown in the inset. The upper right part of the figure depicts the spatial profiles of interferometer modes at the consecutive stages of the setup.

positions $x$ and $x^{\prime}$ is given by

$$
p_{\mathrm{c}}\left(x, x^{\prime} \mid \theta\right)=\sum_{i, j=1,2}\left|\left\langle\operatorname{vac}\left|\hat{a}_{i}(x) \hat{b}_{j}\left(x^{\prime}\right) \hat{U}(\theta)\right| \psi_{\mathcal{D}}\right\rangle\right|^{2}
$$

where the summation over $i=1,2$ stems from tracing over the spectral degree of freedom.

As a concrete example we will take two gaussian modes of width $\sigma$ displaced by $d$,

$$
u(x)=\frac{1}{\sqrt[4]{2 \pi \sigma^{2}}} e^{-(x+d / 2)^{2} / 4 \sigma^{2}}, \quad v(x)=u(x-d)
$$

that can be readily prepared by simple experimental means. In the phase estimation procedure, we will exploit information contained in the relative position $\xi=x-x^{\prime}$ of photons detected in coincidence events. The probability distribution for this variable is given explicitly by

$$
\begin{gathered}
p_{\mathrm{c}}(\xi \mid \theta)=\int \mathrm{d} x^{\prime} p_{\mathrm{c}}\left(x^{\prime}+\xi, x^{\prime} \mid \theta\right) \\
=\frac{1}{2 \sigma \sqrt{\pi}}\left(\cos ^{4} \frac{\theta}{2} e^{-(\xi-d)^{2} / 4 \sigma^{2}}\right. \\
\left.+\sin ^{4} \frac{\theta}{2} e^{-(\xi+d)^{2} / 4 \sigma^{2}}-\frac{1}{2} \mathcal{V} \sin ^{2} \theta e^{-\left(\xi^{2}+d^{2}\right) / 4 \sigma^{2}}\right) .
\end{gathered}
$$

When the relative position of the two photons in coincidence events is available, Fisher information defined in equation (5) becomes enhanced by replacing the first term in the sum with the following integral over $\xi$ :

$$
\frac{1}{p_{c}(\theta)}\left(\frac{\mathrm{d} p_{c}}{\mathrm{~d} \theta}\right)^{2} \rightarrow F_{\mathrm{c}}(\theta)=\int \mathrm{d} \xi \frac{1}{p_{\mathrm{c}}(\xi \mid \theta)}\left(\frac{\partial p_{\mathrm{c}}(\xi \mid \theta)}{\partial \theta}\right)^{2}
$$

In Fig. 1c, we depict the estimation precision for the ratio $d / \sigma=1.64$, which was used in the experiment described below. It 
is seen that the precision is brought to the sub-shot-noise regime over the entire operating range.

Experiment. To verify experimentally sub-shot-noise phase sensitivity of the interferometric scheme described above, we constructed an optical setup shown in Fig. 4. The interferometer is fed with $800 \mathrm{~nm}$ photon pairs generated via type-II spontaneous parametric down-conversion process, which are synchronized using a delayed line, spatially filtered through a single-mode fibre and delivered to the setup in two mutually orthogonal linear polarizations corresponding to the two input ports of the Mach-Zehnder interferometer. The photons emerging from the fibre were partly separated in space by inserting a $1.9-\mathrm{mm}$-long calcite displacer $\mathrm{D}$, which results in the displacement of $d=200 \mu \mathrm{m}$ between the two ortogonally polarized output paths. The spatial modes can be modelled by gaussian functions defined in equation (12) with $\sigma=122 \mu \mathrm{m}$. To ensure temporal stability, the interferometer transformation is implemented in the common path configuration as a half-wave plate (HWP), with the rotation angle equal to quadruple the phase shift $\theta$ between the interferometer arms, followed by a calcite beam separator $S$. The equivalence of this setup with the standard Mach-Zehnder interferometer is evidenced by decomposing the interferometer transformation introduced in equation (1) as

$$
\begin{aligned}
& \left(\begin{array}{ll}
\cos \frac{\theta}{2} & \sin \frac{\theta}{2} \\
\sin \frac{\theta}{2} & -\cos \frac{\theta}{2}
\end{array}\right) \\
& =\left(\begin{array}{cc}
\cos \frac{\theta}{4} & -\sin \frac{\theta}{4} \\
\sin \frac{\theta}{4} & \cos \frac{\theta}{4}
\end{array}\right)\left(\begin{array}{cc}
1 & 0 \\
0 & -1
\end{array}\right)\left(\begin{array}{ll}
\cos \frac{\theta}{4} & \sin \frac{\theta}{4} \\
-\sin \frac{\theta}{4} & \cos \frac{\theta}{4}
\end{array}\right) .
\end{aligned}
$$

In the common path configuration the diagonal matrix with entries 1 and -1 describes the HWP in the coordinate system of its principal axes and the two outer matrices correspond to rotation to the laboratory reference frame by an angle $\theta / 4$.

We determined through an independent measurement (see Methods section) the residual spectral distinguishability of the photons to yield $\mathcal{V}=93 \%$. Note that this value of visibility has been used in the example presented in Fig. 1b. The transverse distance between the two spatially separated modes corresponding to the output ports of the interferometer is $3.2 \mathrm{~mm}$. The rear surface of the separator $\mathrm{S}$ is imaged by means of a spherical lens onto a single-photon-sensitive camera system (see Methods section) capable of spatially resolved detection of coincidence events $^{38,39}$. The profiles of spatial modes propagating in this case through consecutive stages of the setup are shown in the upper right part of Fig. 4.

We recorded spatially resolved two-photon detection events for three values of the phase shift around the dark fringe, $\theta=\pi / 2-0.1, \pi / 2, \pi / 2+0.1$, registering $\sim 6 \times 10^{3}$ events in each case. Experimentally observed spatial distributions $p_{c}\left(x, x^{\prime} \mid \theta\right)$ of coincidence events along with their marginals $p_{c}(\xi \mid \theta)$ for $\xi=x-x^{\prime}$ are presented in Fig. 5. It is seen that the joint position distributions are clearly sensitive to the phase shift $\theta$, in particular, the sign of its deviation from $\pi / 2$ can be unambiguously inferred from the asymmetry of the distribution with respect to the diagonal.

To quantify information about the phase shift present in spatial distributions, we performed phase estimation from the actual experimental data and determined the estimation precision. In the preliminary step, we verified the applicability of equation (13) as a statistical model for the collected data assuming independently measured mode parameters $\sigma$ and $d$. We used the maximum-likelihood method to fit $\mathcal{V}$ and $\theta$, obtaining for the three cases depicted in Fig. 5b respective phase values $\theta=1.47(2)$, $1.57(2), 1.69(2)$ and $\mathcal{V}=0.93$, which is in agreement with the visibility inferred from the independently measured Hong-Ou-Mandel dip.
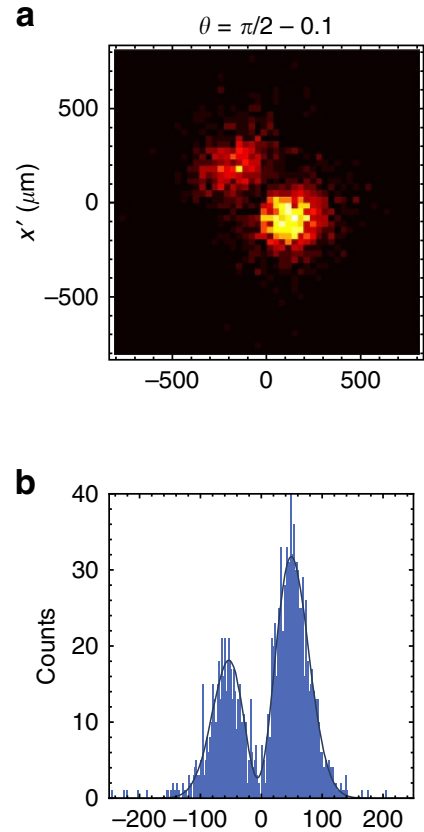
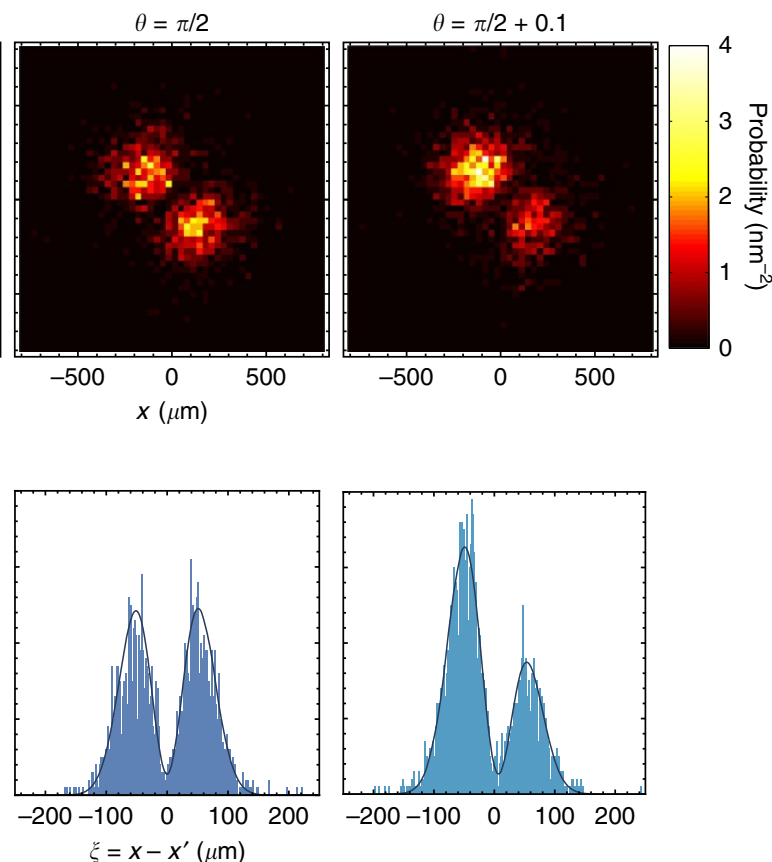

Figure 5 | Spatial distributions of coincidence events. (a) Experimentally measured joint distributions $p_{\mathrm{c}}\left(x, x^{\prime} \mid \theta\right)$ of detecting two photons at positions $x$ and $x^{\prime}$ in two different output ports of the interferometer for photon pairs characterized by spectral indistinguishability $\mathcal{V}=0.93$ and three phase shifts $\theta=\pi / 2-0.1, \pi / 2, \pi / 2+0.1$. (b) Useful information about the phase shift can be extracted from the marginal histograms of the relative position between the photons $\xi=x-x^{\prime}$, shown along with the fitted theoretical model $p_{\mathrm{c}}(\xi \mid \theta)$ used in the estimation procedure (solid lines). 
To determine the actual estimation precision, we divided data obtained for a given HWP setting into $\sim 600$ subsets of 10 two-photon detection events and estimated the value of the phase shift separately from each subset. The width of the resulting distribution of individual estimates can be used as a figure for the estimation precision. The choice of the right estimation procedure needs some attention for small sizes of data sets. The maximum-likelihood estimator is known to be asymptotically efficient ${ }^{40}$, that is, it saturates the Cramér-Rao bound in the asymptotic limit of infinitely many independent data samples. However, its application to small data sets is not justified owing to potential biasedness. Therefore, we used an estimator that is manifestly unbiased for any data size and yields the precision given by equation (4) at least in the vicinity of a given operation point. Specifically, for an experimentally measured statistical frequency distribution $f(\xi)$ of the relative distance between the two photons, this estimator in the vicinity of $\theta_{0}=\pi / 2$ is explicitly given by ${ }^{17}$

$$
\tilde{\theta}[f]=\theta_{0}+\left.\frac{1}{F_{\mathrm{c}}\left(\theta_{0}\right)} \int \mathrm{d} \xi \frac{f(\xi)}{p_{\mathrm{c}}\left(\xi \mid \theta_{0}\right)} \frac{\mathrm{d} p_{\mathrm{c}}\left(\xi \mid \theta_{0}\right)}{\mathrm{d} \theta}\right|_{\theta=\theta_{0}},
$$

where $F_{c}(\theta)$ has been defined in equation (14). In practice, the integral in the above formula is discretized according to the width of the histogram bins. Applying this estimator to individual data subsets yielded a distribution of phase estimates. The estimation precision was determined as the s.d. of this distribution, shown in Fig. 1c for the three phase shifts along with two-sigma error bars. The obtained values, clearly situated below the shot-noise limit, demonstrate quantum-enhanced operation of the interferometer despite partial spectral distinguishability of the input photons.

The analysis presented above was based on processing only the detected sample of photon pairs. This is a routine approach in proof-of-principle experiments employing 'modest-efficiency detectors 2,44 . The detected events are generated by a random subset of all photon pairs produced by the source and therefore provide fair statistics to characterize the multiphoton interference underlying the observed quantum enhancement. Current advances in source and detector technology should enable soon unconditional demonstrations of quantum-enhanced metrology also in more complex scenarios such as the one described here.

\section{Discussion}

We analysed operation of a two-photon Mach-Zehnder interferometer, which is one of the first ${ }^{22}$ and technologically most advanced examples of quantum-enhanced metrology, in a scenario that included two degrees of freedom for interfering photons. It was assumed that one degree of freedom was inaccessible experimentally. Residual distinguishability of interfering photons in this degree of freedom has a markedly deleterious effect on the precision of phase estimation around the operating point where coincidences at the interferometer output are suppressed owing to the Hong-Ou-Mandel effect, producing a coincidence dark fringe. We showed that exploiting another, completely uncorrelated degree of freedom over which one has full experimental control can mitigate this effect, restoring quantum-enhanced precision in the entire operating range. This result is based on a rather subtle interplay between one- and two-photon interference. At the coincidence dark-fringe, imperfect two-photon interference alone does not provide any information about the phase shift, while one-photon interference obviously cannot surpass the shot-noise limit on its own. We demonstrated that combining coherently both types of interference through a suitably designed preparation and measurement scheme in the second degree of freedom yields precision below the shot-noise limit. The feasibility of this approach was confirmed in an experiment using the transverse position of the photons as the controllable degree of freedom that could be measured with high resolution using a single-photon sensitive camera system ${ }^{38,39}$. The presented strategy can be also applied to other scenarios involving two independent degrees of freedom with different experimental accessibility, for example, to mitigate effects of residual spatial distinguishability when only area-integrating detection is available, but photons can be suitably manipulated and measured in the spectral domain.

Beyond two-photon interferometry, one can consider a simple scenario involving a higher photon number when the input ports of a Mach-Zenhder interferometer are fed, respectively, with two photons and a single photon, as shown in Fig. 6a. If all three photons are indistinguishable, counting photons at the interferometer outputs yields fringes depicted in Fig. $6 \mathrm{~b}$ for which Fisher information $F^{(3)}=7$ irrespectively of the operating point. This is more than twofold improvement over the shot-noise limit for three photons used independently. When the single photon exhibits residual spectral distinguishability with respect to the two photons (assumed to be identical) feeding the other port, minute changes of the shapes of the interference fringes have a marked effect around points $\theta_{0}=2 \arctan (1 / \sqrt{2})$ and $\pi-\theta_{0}$ on a relative phase estimation uncertainty $\varepsilon=\Delta^{(3)} / \Delta^{\text {shot }}$ plotted in Fig. 6c, where $\Delta^{(3)}$ is defined analogously to equation (4). According to Fig. $6 \mathrm{~b}$, this can be attributed to a non-vanishing background of events when two photons are detected at one output port and the remaining single photon at the other one. Preparing two photons in the same spatial mode $u(x)$ and the single photon sent individually in a partly overlapping mode $v(x)$ allows one to restore quantum enhancement as seen in Fig. 6c. Interestingly, this effect occurs at different operating points compared with the two-photon case.

A worthwhile candidate to analyse the benefits of mode engineering in a scalable multiphoton scenario may be the celebrated Holland-Burnett scheme ${ }^{45}$ employing two Fock states with equal photon numbers. It is easy to verify that sub-shot-noise precision at $\theta=\pi / 2$ originates from the suppression of odd photon number events at the interferometer outputs, which again is sensitive to residual distinguishability of input photons. A quantitative analysis of this scenario would require developing an efficient approach to deal with multimode multiphoton states. On the other hand, it is known that in the presence of certain common imperfections, such as photon loss, the ultimate precision follows asymptotically the shot-noise type scaling and the quantum enhancement has the form of a multiplicative factor that can be attained via a repetitive use of finite-size multiparticle superposition states ${ }^{46}$. Therefore, results obtained for a fixed number of photons may also prove useful in the asymptotic limit for realistic scenarios.

The presented results are based on the multimode description of a two-photon interferometer, which goes beyond the simplest models typically used to conceive quantum-enhanced measurement schemes. In practice, the applicability of quantum-enhanced techniques depends crucially on the ability to reduce decoherence effects caused by noise and experimental imperfections. Results presented in this paper suggest that in addition to obvious attempts to suppress decoherence effects in interferometry by improving transmission of optical elements, stabilizing phase reference and so on, exploiting the multimode structure of quantum fields can help to achieve the non-classical regime of operation. If the modal structure of the probes is carefully engineered at the input and suitably detected, such a strategy can offer a noticeable improvement in precision even though 
a
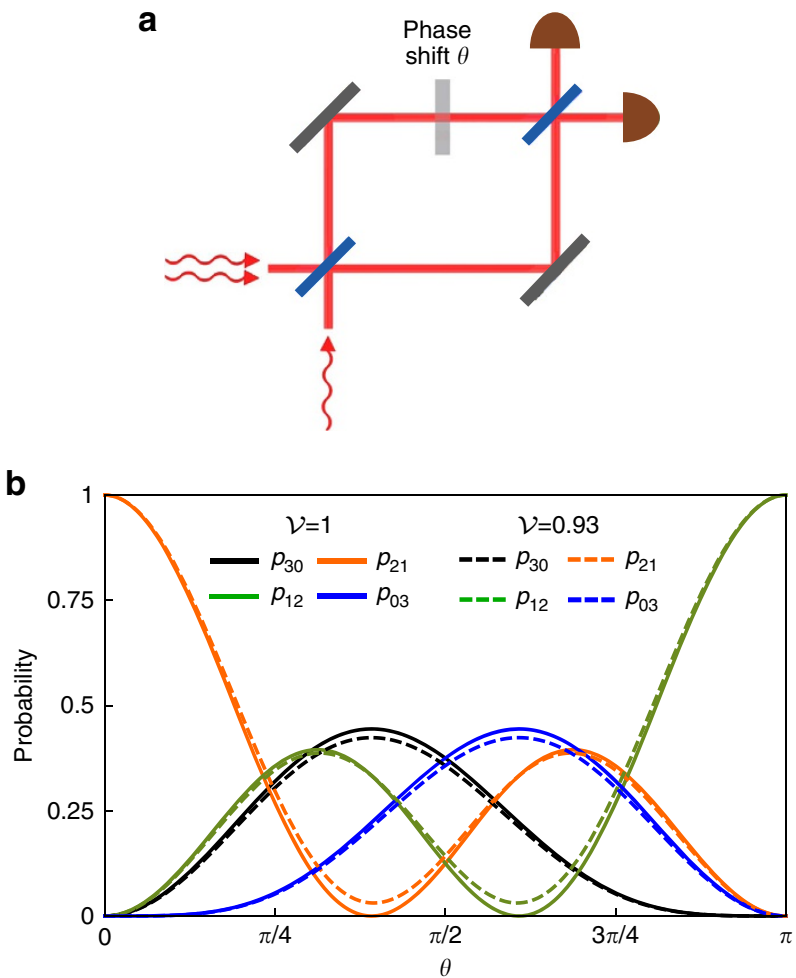

C

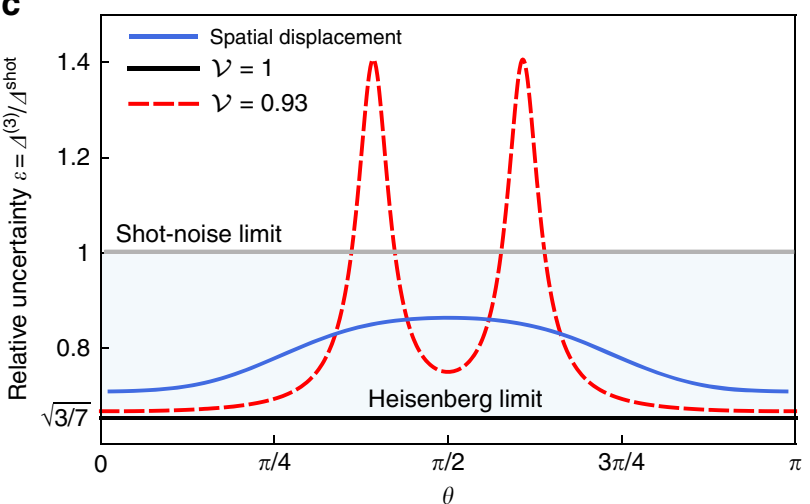

Figure 6 | Three-photon scheme. (a) A Mach-Zehnder interferometer fed with a combination of two photons at one input port and a single photon at the other one. (b) Probabilities $p_{n n^{\prime}}(\theta)$ of detecting, respectively, $n$ and $n^{\prime}$ photons at the interferometer outputs for perfectly indistinguishable photons $\mathcal{V}=1$ and the single photon exhibiting residual distinguishability with respect to the other two mutually identical photons, characterized by $\mathcal{V}=0.93$. (c) In the presence of residual distinguishability, the precision of phase estimation, shown with a dashed red line, deteriorates around operating points where dominant contribution to Fisher information comes from the suppression of events $n n^{\prime}=21$ or 12 . This effect can be mitigated by introducing spatial displacement $d=1.45 \sigma$ between interferometer inputs, resulting in quantum enhancement across the entire operating range (solid blue line). The solid grey line depicts the shot-noise limit of relative uncertainty.

decoherence effects due to another degree of freedom that we do not have control over remain at the same level. An interesting question is whether analogous strategies can be generalized to quantum-enhanced interferometry with squeezed states of light ${ }^{7,41,47}$, boson sampling with linear multiport devices ${ }^{18-21}$ or perhaps benefit other quantum technologies such as optical quantum computing ${ }^{48,49}$.

\section{Methods}

Output two-photon state. For two photons prepared initially in the state $\left|\psi_{\mathcal{D}}\right\rangle$ defined in equation (7) it will be convenient to write the output state as a sum of two components,

$$
\hat{U}(\theta)\left|\psi_{\mathcal{D}}\right\rangle=\left|\psi_{\mathrm{c}}(\theta)\right\rangle+\left|\psi_{\mathrm{d}}(\theta)\right\rangle
$$

Here $\left|\psi_{\mathrm{c}}(\theta)\right\rangle$ is the conditional state describing coincidence events, when the two photons leave the interferometer through different ports

$$
\begin{aligned}
& \left|\psi_{\mathrm{c}}(\theta)\right\rangle=-\left[\sqrt{\mathcal{D} V} \hat{a}_{1 R}^{\dagger} \hat{b}_{1 R}^{\dagger} \cos \theta\right. \\
& +\sqrt{\mathcal{D}(1-\mathcal{V})}\left(\hat{a}_{1 R}^{\dagger} \hat{b}_{2 R}^{\dagger} \cos ^{2} \frac{\theta}{2}-\hat{a}_{2 R}^{\dagger} \hat{b}_{1 R}^{\dagger} \sin ^{2} \frac{\theta}{2}\right) \\
& +\sqrt{(1-\mathcal{D}) \mathcal{V}}\left(\hat{a}_{1 R}^{\dagger} \hat{b}_{1 L}^{\dagger} \cos ^{2} \frac{\theta}{2}-\hat{a}_{1 L}^{\dagger} \hat{b}_{1 R}^{\dagger} \sin ^{2} \frac{\theta}{2}\right) \\
& \left.+\sqrt{(1-\mathcal{D})(1-\mathcal{V})}\left(\hat{a}_{1 R}^{\dagger} \hat{b}_{2 L}^{\dagger} \cos ^{2} \frac{\theta}{2}-\hat{a}_{2 L}^{\dagger} \hat{b}_{1 R}^{\dagger} \sin ^{2} \frac{\theta}{2}\right)\right]|\mathrm{vac}\rangle,
\end{aligned}
$$

while $\left|\psi_{\mathrm{d}}(\theta)\right\rangle$ corresponds to double events, when both the photons emerge at the same output port of the interferometer:

$$
\begin{aligned}
\left|\psi_{\mathrm{d}}(\theta)\right\rangle= & \frac{1}{2} \sin \theta\left(\sqrt{\mathcal{D} \mathcal{V}}\left[\left(\hat{a}_{1 R}^{\dagger}\right)^{2}-\left(\hat{b}_{1 R}^{\dagger}\right)^{2}\right]\right. \\
& +\sqrt{\mathcal{D}(1-\mathcal{V})}\left(\hat{a}_{1 R}^{\dagger} \hat{a}_{2 R}^{\dagger}-\hat{b}_{1 R}^{\dagger} \hat{b}_{2 R}^{\dagger}\right) \\
& +\sqrt{(1-\mathcal{D}) \mathcal{V}}\left(\hat{a}_{1 R}^{\dagger} \hat{a}_{1 L}^{\dagger}-\hat{b}_{1 R}^{\dagger} \hat{b}_{1 L}^{\dagger}\right) \\
& \left.+\sqrt{(1-\mathcal{D})(1-\mathcal{V})}\left(\hat{a}_{1 R}^{\dagger} \hat{a}_{2 L}^{\dagger}-\hat{b}_{1 R}^{\dagger} \hat{b}_{2 L}^{\dagger}\right)\right)|\mathrm{vac}\rangle .
\end{aligned}
$$

The overall probabilities for double and coincidence events are

$$
p_{\mathrm{d}}(\theta)=\left\langle\psi_{\mathrm{d}}(\theta) \mid \psi_{\mathrm{d}}(\theta)\right\rangle=\frac{1}{2}(1+\mathcal{D} \mathcal{V}) \sin ^{2} \theta
$$

and $p_{\mathrm{c}}(\theta)=1-p_{\mathrm{d}}(\theta)$. Inserting $\mathcal{D}=1$ gives as a special case the result presented in equation (3).

Let us note that all the terms in $\left|\psi_{\mathrm{d}}(\theta)\right\rangle$ exhibit identical dependence on $\theta$. Consequently, resolving double events with respect to the spatial degree of freedom cannot yield more information about the phase shift. Therefore, we will focus our attention on coincidence events described by $\left|\psi_{c}(\theta)\right\rangle$. To analyse information about $\theta$ when the spectral degree of freedom cannot be accessed, we will treat it formally as another subsystem $\Omega$, writing $\hat{a}_{i i}^{\dagger} \hat{b}_{i \gamma^{\prime} \gamma^{\prime}}^{\dagger}|\mathrm{vac}\rangle=\left|\chi \chi^{\prime}\right\rangle \otimes\left|i i^{\prime}\right\rangle_{\Omega}$, where $i$, $i^{\prime}=1,2$ and $\chi, \chi^{\prime}=R, L$. Tracing the two-photon state over the spectral subsystem yields the reduced density matrix $\hat{\varrho}_{c}(\theta)=\operatorname{Tr}_{\Omega}\left[\left|\psi_{c}(\theta)\right\rangle\left\langle\psi_{c}(\theta)\right|\right]$, which written in the basis of spatial modes $|R R\rangle,|R L\rangle,|L R\rangle,|L L\rangle$ reads:

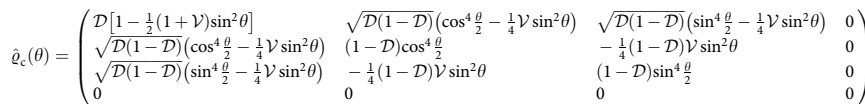

Fisher information. If the spatial degree of freedom of the photons at the interferometer output is projected onto the $R / L$ basis, the complete statistics of measurement results is described by the diagonal elements of the density matrix $\hat{\varrho}_{c}(\theta)$ for coincidence events and the collective probability $p_{\mathrm{d}}(\theta)$ for all double events. An easy calculation yields the corresponding Fisher information

$$
F_{R / L}(\theta)=\frac{2(1+\mathcal{D} \mathcal{V})-(\mathcal{D}+1)(\mathcal{V}+1) \sin ^{2} \theta}{1-\frac{1}{2}(\mathcal{V}+1) \sin ^{2} \theta} .
$$

At the dark fringe we have $F_{R / L}(\pi / 2)=2(1-\mathcal{D})$. This expression, which does not even reach the shot-noise level, can be understood intuitively: information about the phase shift is obtained only from mode-mismatched pairs, when the spatial mode $R$ or $L$ at the output identifies unambiguously the input port of a given photon. The factor $1-\mathcal{D}$ in $F_{R / L}(\pi / 2)$ is the overall fraction of these events, while the constant 2 is contributed by single-photon interference exhibited by such pairs.

Clearly, a measurement in the $R / L$ basis neglects information contained in the off-diagonal elements of the density matrix $\hat{\varrho}_{c}(\theta)$. A measurement strategy that exploits optimally $\hat{\varrho}_{c}(\theta)$ is described by quantum Fisher information involving the symmetric logarithmic derivative. Its calculation is simplified by switching to a new basis for the spatial degree of freedom,

$$
\begin{aligned}
& |\alpha\rangle=\sqrt{\frac{2 \mathcal{D}}{1+\mathcal{D}}}|R R\rangle+\sqrt{\frac{1-\mathcal{D}}{2(1+\mathcal{D})}}(|R L\rangle+|L R\rangle), \\
& |\beta\rangle=\frac{1}{\sqrt{2}}(|R L\rangle-|L R\rangle), \\
& |\gamma\rangle=\sqrt{\frac{1-\mathcal{D}}{1+\mathcal{D}}}|R R\rangle-\sqrt{\frac{\mathcal{D}}{1+\mathcal{D}}}(|R L\rangle+|L R\rangle) .
\end{aligned}
$$


The conditional density matrix $\hat{\varrho}_{c}(\theta)$ takes the form

$$
\begin{aligned}
& \left\langle\alpha\left|\hat{\varrho}_{c}(\theta)\right| \alpha\right\rangle=\frac{1+\mathcal{D}}{2}\left(1-\frac{1+\mathcal{V}}{2} \sin ^{2} \theta\right) \\
& \left\langle\alpha\left|\hat{\varrho}_{c}(\theta)\right| \beta\right\rangle=\left\langle\beta\left|\hat{\varrho}_{c}(\theta)\right| \alpha\right\rangle=\frac{1}{2} \sqrt{1-\mathcal{D}^{2}} \cos \theta \\
& \left\langle\beta\left|\hat{\varrho}_{c}(\theta)\right| \beta\right\rangle=\frac{1-\mathcal{D}}{2}\left(1-\frac{1-\mathcal{V}}{2} \sin ^{2} \theta\right)
\end{aligned}
$$

while all other elements involving $|\gamma\rangle$ or $\langle\gamma|$ vanish. The symmetric logarithmic derivative $\hat{L}_{\mathrm{c}}(\theta)$, given in general by the implicit formula

$$
\frac{\mathrm{d} \hat{\varrho}_{c}}{\mathrm{~d} \theta}=\frac{1}{2}\left[\hat{L}_{\mathrm{c}}(\theta) \hat{\varrho}_{\mathrm{c}}(\theta)+\hat{\varrho}_{\mathrm{c}}(\theta) \hat{L}_{\mathrm{c}}(\theta)\right]
$$

can now be easily found in the two-dimensional subspace spanned by $|\alpha\rangle$ and $|\beta\rangle$. If double events are not resolved in the spatial degree of freedom, quantum Fisher information can be written as

$$
\begin{aligned}
& F_{\mathrm{Q}}(\theta)=\operatorname{Tr}\left\{\hat{\varrho}_{\mathrm{c}}(\theta)\left[\hat{L}_{\mathrm{c}}(\theta)\right]^{2}\right\}+\frac{1}{p_{\mathrm{d}}(\theta)}\left(\frac{\mathrm{d} p_{\mathrm{d}}}{\mathrm{d} \theta}\right)^{2} \\
& =2 \frac{1-\mathcal{D}^{2}+(1+\mathcal{D} \mathcal{V})^{2} \cos ^{2} \theta}{1-\mathcal{D} \mathcal{V}+(1+\mathcal{D} \mathcal{V}) \cos ^{2} \theta} \sin ^{2} \theta+2(1+\mathcal{D} \mathcal{V}) \cos ^{2} \theta
\end{aligned}
$$

where $p_{\mathrm{d}}(\theta)$ is given by equation (20). Specializing the above expression to $\theta=\pi / 2$ yields equation (8), whereas its value for $\mathcal{D}$ optimized individually for a given spectral visibility $0 \leq \mathcal{V} \leq 1$ and an operating point $0 \leq \theta \leq \pi$ is presented in Fig. $2 \mathrm{~b}$. The symmetric logarithmic derivative has a simple off-diagonal form at $\theta=\pi / 2$

$$
\hat{L}_{c}(\pi / 2)=-2 \frac{\sqrt{1-\mathcal{D}^{2}}}{1-\mathcal{D} \mathcal{V}}(|\alpha\rangle\langle\beta|+| \beta\rangle\langle\alpha|)
$$

Quantum Fisher information is saturated by projecting the spatial degree of freedom onto the eigenstates of $\hat{L}_{\mathrm{c}}(\theta)$ (refs 42,43) given explicitly for $\theta=\pi / 2$ by $| \pm\rangle=(|\alpha\rangle \pm|\beta\rangle) / \sqrt{2}$. These states are non-trivial superpositions of photon pairs prepared in combinations of $R, L$ spatial modes at two different output ports of the interferometer.

Experimental details. In the experiment we used a photon pair source based on the II-type SPDC process in a 5-mm-long periodically poled KTP crystal (Raicol Crystals) pumped with $8 \mathrm{~mW}$ of $400 \mathrm{~nm}$ light from a continuous wave diode laser. The produced pairs are transmitted through a $3 \mathrm{~nm}$ full-width at half maximum interference filter, carefully synchronized in time using a delay line and spatially filtered using the single-mode fibre. The gaussian-like spatial modes of the photons after the fibre have a flat phase and the half-width $\sigma=122 \mu \mathrm{m}$ at $1 / e$ height for the intensity distribution measured at the position of the camera system. The residual spectral distinguishability of the photons was determined from the depth of the Hong-Ou-Mandel dip scanned using the delay line and measured with standard avalanche photodiodes for the HWP orientation corresponding to $\theta=\pi / 2$.

Our camera system ${ }^{38,39}$ begins with an image intensifier (Hamamatsu V7090D) where each detected photon that induces a photoelectron emission produces a macroscopic charge avalanche resulting in a bright flash at the output phosphor screen. The flashes are subsequently imaged with a relay lens onto a fast, low-noise $6.5 \times 6.5 \mu \mathrm{m}$ pixel size sCMOS sensor (Andor Zyla) and recorded as $\sim 25$-px gaussian wide spots, which can be easily discriminated from the low-noise background. The central positions of the spots are retrieved from each captured frame with a subpixel resolution by a real-time software algorithm that provides full information about transverse coordinates of each registered coincidence event as illustrated in the inset of Fig. 4. For the sake of simplicity, we consider only the coordinate $x$ in the horizontal plane of the setup and integrate the signals in the vertical direction. A cylindrical lens with $f=30 \mathrm{~mm}$ in front of the detector was used to reduce the vertical size of the image, producing effectively a $700 \times 22 \mathrm{px}$ stripe, which significantly decreases frame readout time and allows us to reach $7 \mathrm{kHz}$ collection rate of frames with exposure time $30 \mathrm{~ns}$ each. The overall quantum efficiency of the camera system is $23 \%$.

Three-photon scheme. A straightforward but tedious calculation shows that for a three-photon input state of the form $\left(\hat{a}_{1 R}^{\dagger}\right)^{2}\left[\sqrt{\mathcal{V}}\left(\sqrt{\mathcal{D}} \hat{b}_{1 R}^{\dagger}+\sqrt{1-\mathcal{D}} \hat{b}_{1 L}^{\dagger}\right)\right.$ $\left.+\sqrt{1-\mathcal{V}}\left(\sqrt{\mathcal{D}} \hat{b}_{2 R}^{\dagger}+\sqrt{1-\mathcal{D}} \hat{b}_{2 L}^{\dagger}\right)\right] \mid$ vac $\rangle$ spatially resolved distributions for events 21 and 12 when the photons are split between the interferometer output ports into two and one are given by

$$
\begin{aligned}
& p_{21}\left(x_{1}, x_{2} ; x^{\prime} \mid \theta\right)=\mathcal{V} \mid \frac{1}{2} \sin \theta \sin \frac{\theta}{2}\left(u\left(x_{1}\right) v\left(x_{2}\right)\right. \\
& \left.\quad+u\left(x_{2}\right) v\left(x_{1}\right)\right) u\left(x^{\prime}\right)-\left.\cos ^{3} \frac{\theta}{2} u\left(x_{1}\right) u\left(x_{2}\right) v\left(x^{\prime}\right)\right|^{2} \\
& +(1-\mathcal{V})\left[\frac{1}{4} \sin ^{2} \theta \sin ^{2} \frac{\theta}{2}\left(\left|u\left(x_{1}\right)\right|^{2}\left|v\left(x_{2}\right)\right|^{2}+\left|v\left(x_{1}\right)\right|^{2}\left|u\left(x_{2}\right)\right|^{2}\right)\right. \\
& \left.\quad \times\left|u\left(x^{\prime}\right)\right|^{2}+\cos ^{6} \frac{\theta}{2}\left|u\left(x_{1}\right)\right|^{2}\left|u\left(x_{2}\right)\right|^{2}\left|v\left(x^{\prime}\right)\right|^{2}\right]
\end{aligned}
$$

and

$$
\begin{aligned}
& p_{12}\left(x ; x_{1}^{\prime}, x_{2}^{\prime} \mid \theta\right)=\mathcal{V} \mid \sin ^{3} \frac{\theta}{2} v(x) u\left(x_{1}^{\prime}\right) u\left(x_{2}^{\prime}\right) \\
& -\left.\frac{1}{2} \sin \theta \cos \frac{\theta}{2} u(x)\left(u\left(x_{1}^{\prime}\right) v\left(x_{2}^{\prime}\right)+u\left(x_{2}^{\prime}\right) v\left(x_{1}^{\prime}\right)\right)\right|^{2} \\
& +(1-\mathcal{V})\left[\frac { 1 } { 4 } \operatorname { s i n } ^ { 2 } \theta \operatorname { c o s } ^ { 2 } \frac { \theta } { 2 } | u ( x ) | ^ { 2 } \left(\left|u\left(x_{1}^{\prime}\right)\right|^{2}\left|v\left(x_{2}^{\prime}\right)\right|^{2}\right.\right. \\
& \left.+\left|u\left(x_{2}^{\prime}\right)\right|^{2}\left|v\left(x_{1}^{\prime}\right)\right|^{2}+\sin ^{6} \frac{\theta}{2}|v(x)|^{2}\left|u\left(x_{1}^{\prime}\right)\right|^{2}\left|u\left(x_{2}^{\prime}\right)\right|^{2}\right]
\end{aligned}
$$

where we have made use of equation (10). Fisher information taking into account spatially resolved detection of events 21 and 12 is given by

$$
\begin{aligned}
& F^{(3)}(\theta)=\frac{1}{p_{30}(\theta)}\left(\frac{\mathrm{d} p_{30}}{\mathrm{~d} \theta}\right)^{2}+\frac{1}{p_{03}(\theta)}\left(\frac{\mathrm{d} p_{03}}{\mathrm{~d} \theta}\right)^{2} \\
& +\int \frac{\mathrm{d} x_{1} \mathrm{~d} x_{2} \mathrm{~d} x^{\prime}}{p_{21}\left(x_{1}, x_{2} ; x^{\prime} \mid \theta\right)}\left(\frac{\partial}{\partial \theta} p_{21}\left(x_{1}, x_{2} ; x^{\prime} \mid \theta\right)\right)^{2} \\
& +\int \frac{\mathrm{d} x \mathrm{~d} x_{1}^{\prime} \mathrm{d} x_{2}^{\prime}}{p_{12}\left(x ; x_{1}^{\prime}, x_{2}^{\prime} \mid \theta\right)}\left(\frac{\partial}{\partial \theta} p_{12}\left(x ; x_{1}^{\prime}, x_{2}^{\prime} \mid \theta\right)\right)^{2} .
\end{aligned}
$$

Assuming Gaussian spatial modes introduced in equation (12), the above expression was optimized over the displacement $d$ for $\theta_{0}=2 \arctan (1 / \sqrt{2})$ and $\mathcal{V}=93 \%$. The relative uncertainty of a phase estimate for the obtained value $d=1.45 \sigma$ has been depicted for the entire range $0 \leq \theta \leq \pi$ in Fig. $6 c$.

\section{References}

1. Brida, G., Genovese, M. \& Ruo Berchera, I. Experimental realization of sub-shot-noise quantum imaging. Nat. Photon. 4, 227-230 (2010).

2. Ono, T., Okamoto, R. \& Takeuchi, S. An entanglement-enhanced microscope. Nat. Commun. 4, 2426 (2013).

3. Schwartz, O. et al. Superresolution microscopy with quantum emitters. Nano Lett. 13, 5832-5836 (2013).

4. Tsang, M. Quantum imaging beyond the diffraction limit by optical centroid measurements. Phys. Rev. Lett. 102, 253601 (2009).

5. Wolfgramm, F., Vitelli, C., Beduini, F. A., Godbout, N. \& Mitchell, M. W. Entanglement-enhanced probing of a delicate material system. Nat. Photon. 7, 28-32 (2012).

6. Crespi, A. et al. Measuring protein concentration with entangled photons. Appl. Phys. Lett. 100, 233704 (2012).

7. LIGO Collaboration. Enhanced sensitivity of the LIGO gravitational wave detector by using squeezed states of light. Nat. Photon. 7, 613-619 (2013).

8. Caves, C. M. Quantum-mechanical noise in an interferometer. Phys. Rev. D 23, 1693-1708 (1981).

9. Yurke, B., McCall, S. L. \& Klauder, J. R. SU(2) and SU(1,1) interferometers. Phys. Rev. A 33, 4033-4054 (1986).

10. Bollinger, J. J., Itano, W. M., Wineland, D. J. \& Heinzen, D. J. Optimal frequency measurements with maximally correlated states. Phys. Rev. A 54, R4649-R4652 (1996).

11. Boto, A. N. et al. Quantum interferometric optical lithography: exploiting entanglement to beat the diffraction limit. Phys. Rev. Lett. 85, 2733-2736 (2000).

12. Giovannetti, V., Lloyd, S. \& Maccone, L. Quantum metrology. Phys. Rev. Lett. 96, 10401 (2006).

13. Paris, M. G. A. Quantum estimation for quantum technology. Int. J. Quantum Inf. 7, 125 (2009).

14. Banaszek, K., Demkowicz-Dobrzański, R. \& Walmsley, I. A. Quantum states made to measure. Nat. Photon. 3, 673-676 (2009).

15. Maccone, L. \& Giovannetti, V. Quantum metrology: Beauty and the noisy beast. Nat. Phys. 7, 376-377 (2011).

16. Tóth, G. \& Apellaniz, I. Quantum metrology from a quantum information science perspective. J. Phys. A 47, 424006 (2014).

17. Demkowicz-Dobrzański, R., Jarzyna, M. \& Kołodyński, J. Quantum limits in optical interferometry. Prog. Optics 60, 345-435 (2015)

18. Spring, J. B. et al. Boson sampling on a photonic chip. Science 339, 798-801 (2013). 
19. Spagnolo, N. et al. Experimental validation of photonic boson sampling. Nat. Photon. 8, 615-620 (2014).

20. Tichy, M. C., Mayer, K., Buchleitner, A. \& Mølmer, K. Stringent and efficient assessment of boson-sampling devices. Phys. Rev. Lett. 113, 020502 (2014).

21. Motes, K. R., Gilchrist, A., Dowling, J. P. \& Rohde, P. P. Scalable boson sampling with time-bin encoding using a loop-based architecture. Phys. Rev. Lett. 113, 120501 (2014).

22. Rarity, J. G. et al. Two-photon interference in a Mach-Zehnder interferometer. Phys. Rev. Lett. 65, 1348-1351 (1990).

23. Huelga, S. F. et al. Improvement of frequency standards with quantum entanglement. Phys. Rev. Lett. 79, 3865-3868 (1997).

24. Dorner, U. et al. Optimal quantum phase estimation. Phys. Rev. Lett. 102, 40403 (2009).

25. Knysh, S., Smelyanskiy, V. N. \& Durkin, G. A. Scaling laws for precision in quantum interferometry and the bifurcation landscape of the optimal state. Phys. Rev. A 83, 21804 (2011).

26. Jiang, K. et al. Strategies for choosing path-entangled number states for optimal robust quantum-optical metrology in the presence of loss. Phys. Rev. A 86, 013826 (2012).

27. Datta, A. et al. Quantum metrology with imperfect states and detectors. Phys. Rev. A 83, 63836 (2011)

28. Escher, B. M., de Matos Filho, R. L. \& Davidovich, L. General framework for estimating the ultimate precision limit in noisy quantum-enhanced metrology. Nat. Phys. 7, 406-411 (2011).

29. Demkowicz-Dobrzański, R., Guta, M. \& Kołodyński, J. The elusive Heisenberg limit in quantum enhanced metrology. Nat. Commun. 3, 1063 (2012).

30. Peeters, W., Renema, J. \& van Exter, M. Engineering of two-photon spatial quantum correlations behind a double slit. Phys. Rev. A 79, 043817 (2009).

31. Shin, H., Chan, K. W. C., Chang, H. J. \& Boyd, R. W. Quantum spatial superresolution by optical centroid measurements. Phys. Rev. Lett. 107, 083603 (2011).

32. Rozema, L. A. et al. Scalable spatial superresolution using entangled photons. Phys. Rev. Lett. 112, 223602 (2014).

33. Abouraddy, A. F., Nasr, M. B., Saleh, B. E. A., Sergienko, A. V. \& Teich, M. C. Demonstration of the complementarity of one- and two-photon interference. Phys. Rev. A 63, 63803 (2001).

34. Edgar, M. P. et al. Imaging high-dimensional spatial entanglement with a camera. Nat. Commun. 3, 984 (2012).

35. Moreau, P.-A., Devaux, F. \& Lantz, E. Einstein-Podolsky-Rosen paradox in twin images. Phys. Rev. Lett. 113, 160401 (2014).

36. Lemos, G. B. et al. Quantum imaging with undetected photons. Nature $\mathbf{5 1 2}$ 409-412 (2014)

37. Morris, P. A., Aspden, R. S., Bell, J. E. C., Boyd, R. W. \& Padgett, M. J. Imaging with a small number of photons. Nat. Commun. 6, 5913 (2015).

38. Chrapkiewicz, R., Wasilewski, W. \& Banaszek, K. High-fidelity spatially resolved multiphoton counting for quantum imaging applications. Opt. Lett. 39, 5090-5093 (2014).

39. Jachura, M. \& Chrapkiewicz, R. Shot-by-shot imaging of Hong-Ou-Mandel interference with an intensified sCMOS camera. Opt. Lett. 40, 1540-1543 (2015).

40. Kay, S. M. Fundamentals of Statistical Signal Processing: Estimation Theory (Prentice Hall, 1993).
41. Demkowicz-Dobrzański, R., Banaszek, K. \& Schnabel, R. Fundamental quantum interferometry bound for the squeezed-light-enhanced gravitational wave detector GEO 600. Phys. Rev. A 88, 41802 (2013).

42. Helstrom, C. W. Quantum Detection and Estimation Theory (Academic press, 1976).

43. Braunstein, S. L. \& Caves, C. M. Statistical distance and the geometry of quantum states. Phys. Rev. Lett. 72, 3439-3443 (1994).

44. Nagata, T. L., Okamoto, R., O’Brien, J. L., Sasaki, K. \& Takeuchi, S. Beating the standard quantum limit with four-entangled photons. Science 316, 726-729 (2007).

45. Holland, M. J. \& Burnett, K. Interferometric detection of optical phase shifts at the Heisenberg limit. Phys. Rev. Lett. 71, 1355-1358 (1993).

46. Jarzyna, M. \& Demkowicz-Dobrzański, R. True precision limits in quantum metrology. New J. Phys. 17, 013010 (2015).

47. Afek, I., Ambar, O. \& Silberberg, Y. High-NOON states by mixing quantum and classical light. Science 328, 879-881 (2010).

48. Knill, E., Laflamme, R. \& Milburn, G. J. A scheme for efficient quantum computation with linear optics. Nature 409, 46-52 (2001).

49. Yao, X. C. et al. Experimental demonstration of topological error correction. Nature 482, 489-494 (2012).

\section{Acknowledgements}

We acknowledge inspiring discussion with M.G. Raymer and I.A. Walmsley. This project was financed by the National Science Centre, No. DEC-2013/09/N/ST2/02229, DEC2011/03/D/ST2/01941, Polish Ministry of Science and Higher Education Iuventus Plus program for years 2015-2017, No. 0088/IP3/2015/73, European Commission under the FP7 projects SIQS (Grant Agreement No. 600645) and PhoQuS@UW (Grant Agreement No. 316244) co-financed by the Polish Ministry of Science and Higher Education. R.C. was supported by Foundation for Polish Science (FNP).

\section{Author contributions}

M.J., R.C. and W.W. designed and performed the experiment. R.D.D. developed theory and analysed the experimental data. K.B. conceived the scheme and interpreted results. All authors contributed to the writing of the manuscript

\section{Additional information}

Competing financial interests: The authors declare no competing financial interests.

Reprints and permission information is available online at http://npg.nature.com/ reprintsandpermissions/

How to cite this article: Jachura, M. et al. Mode engineering for realistic quantum-enhanced interferometry. Nat. Commun. 7:11411 doi: 10.1038/ncomms11411 (2016)

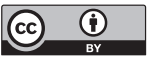

This work is licensed under a Creative Commons Attribution 4.0 International License. The images or other third party material in this article are included in the article's Creative Commons license, unless indicated otherwise in the credit line; if the material is not included under the Creative Commons license, users will need to obtain permission from the license holder to reproduce the material. To view a copy of this license, visit http://creativecommons.org/licenses/by/4.0/ 